\title{
La explotación del vacuno ecológico en sistemas de dehesas del SO de España: Análisis de parámetros técnicos
}

\author{
Horrillo, A. ' ; Mesías, F.J. ${ }^{2 @ ; ~ G a s p a r, ~ P . ~}{ }^{3}$ y Escribano, M. ${ }^{1}$
}

\begin{abstract}
'Dpto. Producción Animal y Ciencia de los Alimentos. Facultad de Veterinaria. Universidad de Extremadura. Cáceres. España. ${ }^{2}$ Dpto. Economía. Escuela de Ingenierías Agrarias. Universidad de Extremadura. Badajoz. España.

${ }^{3}$ Dpto. Producción Animal y Ciencia de los Alimentos. Escuela de Ingenierías Agrarias. Universidad de Extremadura. Badajoz. España.
\end{abstract}

PALABRAS CLAVE ADICIONALES

Producción ecológica.

Caracterización.

Sistemas extensivos.

\section{RESUMEN}

La producción de bovino ecológico en dehesas supone unos de los grandes retos de estudio en los sistemas de producción extensivos. En este sentido, el trabajo analiza una muestra de 34 explotaciones de vacuno ecológico (55,7\% del total) de la provincia de Badajoz a través de indicadores de gestión técnica que nos permita abordar su caracterización. Los indicadores utilizados en el trabajo nos aportan información sobre la estructura y las características de las explotaciones analizadas en relación a los usos del suelo y presión ganadera, rendimientos técnicos y mano de obra. La conversión de los sistemas vacunos extensivos a la producción ecológica se ve facilitada por la similitud de los indicadores técnicos entre ambos tipos de explotaciones.

Organic beef farms in the dehesa systems of Spain: Analysis of technical indicators SUMMARY

ADDITIONAL KEYWORDS

Organic farming.

Characterization.

Extensive systems.

INFORMACIÓN

Cronología del artículo.

Recibido/Received: 10.2.2015

Aceptado/Accepted: 25.5.2015

On-line: 16.9.2015

Correspondencia a los autores/Contact e-mail:

fimesias@unex.es

\section{INTRODUCCIÓN}

Los sistemas de producción animal en áreas de dehesas destacan por su alto valor medioambiental y socioeconómico (Escribano et al., 2001) y en ellos la actividad ganadera juega un papel esencial en su mantenimiento y conservación. La multifuncionalidad de las explotaciones y el uso diversificado de los recursos por las diferentes especies ganaderas son aspectos relevantes en su gestión (Gaspar et al., 2007), en la que el ganado bovino juega un papel determinante.

La agricultura ecológica en España ha venido experimentando un importante auge en los últimos años. Extremadura cuenta actualmente con 42.558 hectáreas de pastos inscritos como ecológicos, con un total de 162 ganaderías de las cuales 95 son de ganado bovino ecológico (MAGRAMA, 2013). No obstante, la adaptación no es igual en todas las especies ganaderas y sistemas de explotación (Blanco-Penedo et al., 2012). La escasa rentabilidad de las explotaciones ganaderas de dehesa ha obligado a los productores a realizar cambios adaptativos en su modelo (Gaspar et al., 2009) lo que ha contribuido a un incremento de las explotaciones ecológicas de vacuno de carne.

En este contexto, el trabajo analiza una muestra de las explotaciones de bovino ecológico en la provincia de Badajoz (Extremadura) a través de indicadores de gestión técnica que nos permita abordar una caracterización de estos sistemas.

\section{MATERIAL Y MÉTODOS}

El trabajo analiza una muestra de 34 explotaciones de vacuno ecológico en extensivo de las 61 existentes en la provincia de Badajoz dentro del proyecto INIA (RTA2009-00122-C03-03). Se seleccionaron aquellas en 
las que su producción principal fuera el ganado vacuno ecológico, bien explotado como una única especie o junto con ovino y/o porcino. De las explotaciones analizadas, 12 eran fincas donde se explotaba exclusivamente bovino ecológico, 12 explotaciones ganaderas mixtas donde se abordaban procesos de cebo y 10 explotaciones mixtas de pequeña dimensión territorial.

Los datos para la realización del estudio se obtuvieron mediante encuesta directa al titular de la explotación en el año 2011.

Partiendo de la información derivada de la encuesta se establecieron diferentes indicadores elaborados a partir de la bibliografía existente (Escribano et al. ,2001; Milán et al., 2003; Gaspar et al., 2007 y 2009), así como por la experiencia en trabajos anteriores de los autores.

Los indicadores utilizados en el trabajo nos aportan información sobre la estructura y las características de las explotaciones analizadas. Estos se integran en tres bloques: Usos del suelo y presión ganadera, productividad numérica y parámetros técnicos y Mano de obra. El tratamiento y análisis de la información se realizó con el paquete estadístico SPSS (v.18).

\section{RESULTADOS Y DISCUSIÓN}

La tabla I contiene los resultados obtenidos en relación a los usos del suelo, rendimientos técnicos (incluido cebo) y mano de obra. Se observa que las explotaciones presentan una extensión media de 278,15 hectáreas, frecuente en las explotaciones de ganado vacuno en Extremadura (Gaspar et al., 2007), con un régimen en propiedad del 47,40\% de su superficie.

Las explotaciones analizadas presentan un alto porcentaje de superficie arbolada (75\%) y las tierras de cultivo están presentes en un 8,81\% de su superficie, con lo que más de dos tercios de la SAU se dedica exclusivamente a pastoreo.

La dimensión media por explotación es de 83,38 UGM de ganado bovino, con una carga ganadera media de 0,35 UGM/ha de las 0,41 UGM/ha de carga ganadera total. Estos resultados coinciden con los encontrados en explotaciones situadas en dehesas del suroeste español (Escribano et al., 2001) y con explotaciones tradicionales de dehesas analizadas por otros autores (Plieringer y Wilbrand, 2001).

En relación a los rendimientos ganaderos se observa que la tasa de reposición (12,08\%), el número de vacas/toro $(28,32)$, los terneros nacidos por vaca $(0,8)$, los terneros destetados $(0,76)$ y la edad de destete (de 5,94 meses y con un peso de medio de 189,71 kg) concuerdan con los resultados encontrados tanto en explotaciones de vacuno convencional como ecológico en dehesas (Martín et al., 2001; Plieninger y Wilbrand, 2001; Milán et al., 2006; Perea et al., 2007).

Sin embargo y como rasgo de las explotaciones de bovino ecológico se observa un mayor incremento de las tasas de mortalidad traducido en un menor nivel de terneros destetados, con respecto a los nacidos (94,39\%), así como la baja capacidad de cebo de las explotaciones ya que tan sólo son vendidos como añojos 11 animales por cada 100 vacas. Este hecho unido a la falta de cebaderos especializados supone uno de los principales retos de la producción ecológica en sistemas extensivos como la dehesa y deja entrever una inadecuada comercialización de sus producciones. De las explotaciones que ceban destaca un peso final de 493 $\mathrm{kg}$, algo inferior al tradicional. El resto de parámetros es similar al encontrado en las explotaciones con cebo de bovino en dehesas (Martín et al., 2001).

Del análisis de la mano de obra se desprende que la presencia media de trabajadores es de 1 UTA por

Tabla I. Características técnicas de las explotaciones de bovino ecológico en Badajoz (Extremadura) (Technical indicators of organic beef farms in Badajoz (Extremadura)).

\begin{tabular}{|c|c|c|c|}
\hline INDICADORES DE USOS DEL SUELO Y CARGAS GANADERAS & Media \pm ES & INDICADORES DE RENDIMIENTOS & Media $\pm E S$ \\
\hline Superficie total (ha) & $278,15 \pm 29,14$ & $\mathrm{~N}^{\circ}$ vacas/toro & $28,32 \pm 2,33$ \\
\hline Superficie agraria Útil (ha) & $272,59 \pm 28,55$ & Tasa de reposición (\%) & $12,08 \pm 1,93$ \\
\hline Superficie en propiedad/total (\%) & $47,40 \pm 7,93$ & Terneros nacidos por vaca & $0,80 \pm 0,02$ \\
\hline Superficie arrendada/total (\%) & $52,60 \pm 7,93$ & Terneros destetados por vaca & $0,76 \pm 0,03$ \\
\hline Superficie arbolada/total (\%) & $75,00 \pm 6,00$ & Terneros vendidos por vaca & $0,61 \pm 0,05$ \\
\hline Superficie de explotación cultivad $(\%)^{*}$ & $8,81 \pm 1,99$ & Añojos vendidos por vaca & $0,11 \pm 0,04$ \\
\hline UGM vacuno (UGM) & $83,38 \pm 7,72$ & Fertilidad del vacuno (\%) & $80,92 \pm 2,26$ \\
\hline UGM totales (UGM) & $100,24 \pm 10,27$ & Peso destete vacuno (kg) & $189,71 \pm 2,73$ \\
\hline UGM vacuno/total (UGMvac/UGMtot) & $0,87 \pm 0,03$ & Edad destete terneros (meses) & $5,94 \pm 0,14$ \\
\hline Carga ganadera total(UGMtot/SAU) & $0,41 \pm 0,03$ & Terneros destetados/nacidos (\%) & $94,39 \pm 1,98$ \\
\hline \multirow[t]{2}{*}{ Carga ganadera vacuno (UGMvac/SAU) } & $0,35 \pm 0,02$ & Vacas autóctonas (\%) & $27,32 \pm 6,92$ \\
\hline & & Vacas cruzadas (\%) & $63,39 \pm 6,89$ \\
\hline INDICADORES DE MANO DE OBRA & & INDICADORES DE CEBO & \\
\hline UTA (Unidades trabajo-año) totales & $1,55 \pm 0,11$ & Peso inicio cebo $(\mathrm{kg})$ & $208,75 \pm 3,86$ \\
\hline UTA totales/100 ha SAU & $0,75 \pm 0,07$ & Periodo de cebo (días) & $197,88 \pm 34,78$ \\
\hline UTA fijo/100 ha SAU & $0,14 \pm 0,36$ & Edad inicio cebo (meses) & $6,06 \pm 0,47$ \\
\hline UTA eventual/100 ha SAU & $0,05 \pm 0,02$ & Edad final cebo (meses) & $13,25 \pm 0,40$ \\
\hline UTA familiar/100 ha SAU & $0,56 \pm 0,08$ & Peso final $(\mathrm{kg})$ & $493,13 \pm 14,32$ \\
\hline Unidades trabajo-año/100 UGM & $1,84 \pm 0,13$ & & \\
\hline
\end{tabular}


cada 54 UGM, o bien, 1 UTA por cada 133 ha de SAU. Este indicador dependerá de la carga ganadera, de la superficie total y de la orientación ganadera de la explotación. Sin embargo, se aprecia el gran peso de la mano de obra familiar (un $74,7 \%$ ) lo que es común en explotaciones de bovino ecológicas (Perea et al., 2007) y difiere de los resultados encontrados en explotaciones de dehesa de bovino convencional (Milán et al., 2006).

Se puede concluir que, en términos generales, los indicadores técnicos en las explotaciones analizadas se comportan de manera similar a los de las explotaciones de bovino de carne convencionales. Este hecho facilita, al menos desde el punto de vista técnico, la conversión de los sistemas extensivos a este modelo de producción aunque un enfoque más amplio en el que se tuvieran en cuenta aspectos de manejo y su influencia en la conservación de las dehesas seria de interés para una adecuada implementación de políticas públicas.

\section{AGRADECIMIENTOS}

Los autores agradecen el apoyo y la financiación proporcionada por INIA, Gobierno de Extremadura y FEDER a través del proyecto de investigación RTA2009-00122-C03-03.

\section{BIBLIOGRAFÍA}

Blanco-Penedo, I.; López-Alonso, M.; Shore, R.F.; Miranda, M.; Castillo, C.; Hernández, J. and Benedito, J.L. 2012. Evaluation of organic, conventional and intensive beef farm systems: health, management and animal production. Animal, 6:1503-1511.

Escribano, M.; Rodríguez, A.; Mesías, F.J. y Pulido, F. 2001. Tipologías de sistemas adehesados. Arch Zootec, 50: 411-414.

Gaspar, P.; Mesías, F.J.; Escribano, M.; Rodríguez de Ledesma, A. and Pulido, F. 2007. Economic and management characterization of dehesa farms: Implications for their sustainability. AgroforestSyst, 71: 151-162.

Gaspar, P.; Escribano, M.; Mesías, F.J. and Pulido, F. 2009. Sustainability in Spanish extensive farms (Dehesa): an economic and management indicator-based evaluation. Rangeland Ecol Manag, 62: 153-162.

MAGRAMA. 2013. Datos de estructura bovino de carne. Secretaría G. Técnica. Ministerio de Agricultura, Alimentación, Medio Ambiente.

Martín, M.; Escribano, M.; Mesías, F.J.; Rodríguez, A. y Pulido, F. 2001. Sistemas extensivos de producción animal. Arch Zootec, 50: 465-489.

Milán, M.J.; Bartolomé, J.; Quintanilla, R.; García-Cachan, M.D.; Espejo, M.; Herráiz, P.L.; Sánchez-Recio, J.M. and Piedrafita, J. 2006. Structural characterisation and typology of beef cattle farms of Spain wooded rangelands (dehesas). Livest Sci, 99: 197-209.

Perea, J.; García, A.; Acero, R.; Valerio, D. y V. Rodríguez. 2007. Characterisation of organic beef cattle farms in Andalusia. Arch Zootec, 56: 517-521.

Plieninger, T. and Wilbrand, C. 2001. Land use, biodiversity conservation, and rural development in the dehesas of Cuatro Lugares, Spain. Agroforest Syst, 51: 23-34. 
